\title{
Polar Effects and Structural Variation in 4-Substituted 1-Phenylbicyclo[2.2.2] octane Derivatives: A Quantum Chemical Study
}

By Anna Rita Campanelli, Aldo Domenicano,* and Fabio Ramondo

\section{SUPPORTING INFORMATION}


TABLE S1: Selected Geometrical Parameters of the Benzene Ring in Molecular Systems 1-4, from HF/6-31G* Calculations ${ }^{a, b}$

\begin{tabular}{|c|c|c|c|c|c|c|}
\hline $\begin{array}{l}\text { molecular } \\
\text { system }\end{array}$ & substituent & $\alpha$ & $\beta$ & $\gamma$ & $\delta$ & $a$ \\
\hline \multirow[t]{21}{*}{1} & $\mathrm{O}^{-}$ & $116.10_{8}$ & $122.09_{3}$ & $120.61_{1}$ & $118.48_{1}$ & 1.3970 \\
\hline & $\mathrm{BH}_{3}^{-}$ & $116.20_{3}$ & $122.04_{0}$ & $120.59_{6}$ & $118.52_{4}$ & 1.3967 \\
\hline & $\mathrm{COO}^{-}$ & $116.31_{0}$ & $121.98_{1}$ & $120.57_{8}$ & $118.57_{1}$ & 1.3963 \\
\hline & $\mathrm{MgCl}$ & $116.69_{6}$ & $121.76_{6}$ & $120.50_{3}$ & $118.76_{2}$ & 1.3951 \\
\hline & $\mathrm{H}$ & $116.69_{5}$ & $121.76_{5}$ & $120.50_{5}$ & $118.76_{1}$ & 1.3951 \\
\hline & $\mathrm{Me}$ & $116.71_{3}$ & $121.75_{6}$ & $120.50_{2}$ & $118.76_{8}$ & 1.3951 \\
\hline & $\mathrm{SiH}_{3}$ & $116.72_{3}$ & $121.74_{9}$ & $120.50_{0}$ & $118.77_{5}$ & 1.3951 \\
\hline & $\mathrm{OMe}$ & $116.73_{2}$ & $121.74_{5}$ & $120.50_{0}$ & $118.77_{6}$ & 1.3950 \\
\hline & $\mathrm{NH}_{2}$ & $116.73_{8}$ & $121.74_{2}$ & $120.49_{9}$ & $118.77_{8}$ & 1.3950 \\
\hline & $\mathrm{OH}$ & $116.75_{1}$ & $121.73_{4}$ & $120.49_{5}$ & $118.78_{7}$ & 1.3950 \\
\hline & $\mathrm{COMe}$ & $116.75_{6}$ & $121.73_{1}$ & $120.49_{7}$ & $118.78_{7}$ & 1.3950 \\
\hline & $\mathrm{F}$ & $116.79_{8}$ & $121.70_{8}$ & 120.489 & $118.80_{4}$ & 1.3948 \\
\hline & $\mathrm{Cl}$ & $116.81_{6}$ & $121.69_{8}$ & $120.48_{6}$ & $118.81_{3}$ & 1.3948 \\
\hline & $\mathrm{CF}_{3}$ & $116.81_{4}$ & $121.69_{8}$ & $120.48_{7}$ & $118.81_{4}$ & 1.3948 \\
\hline & $\mathrm{COCl}$ & $116.82_{9}$ & 121.689 & $120.48_{6}$ & $118.81_{8}$ & 1.3948 \\
\hline & $\mathrm{CN}$ & $116.84_{5}$ & $121.67_{9}$ & $120.48_{4}$ & $118.82_{5}$ & 1.3947 \\
\hline & $\mathrm{NO}_{2}$ & $116.87_{1}$ & $121.66_{5}$ & $120.47_{9}$ & $118.83_{7}$ & 1.3947 \\
\hline & $\mathrm{ClO}_{3}$ & $116.95_{9}$ & $121.61_{4}$ & $120.46_{7}$ & $118.87_{7}$ & 1.3945 \\
\hline & $\mathrm{PH}_{3}^{+}$ & $117.23_{3}$ & $121.45_{4}$ & $120.42_{5}$ & $119.00_{4}$ & 1.3940 \\
\hline & $\mathrm{NH}_{3}^{+}$ & $117.26_{8}$ & $121.43_{4}$ & $120.42_{1}$ & $119.01_{9}$ & 1.3940 \\
\hline & $\mathrm{OH}_{2}^{+}$ & $117.34_{1}$ & $121.39_{1}$ & $120.41_{1}$ & $119.05_{1}$ & 1.3939 \\
\hline \multirow[t]{8}{*}{2} & $\mathrm{O}^{-}$ & $116.33_{9}$ & $121.95_{7}$ & $120.59_{5}$ & $118.55_{8}$ & 1.3966 \\
\hline & $\mathrm{BH}_{3}^{-}$ & $116.39_{7}$ & $121.92_{4}$ & $120.58_{4}$ & $118.58_{5}$ & 1.3964 \\
\hline & $\mathrm{COO}^{-}$ & $116.52_{4}$ & $121.85_{5}$ & $120.56_{3}$ & $118.64_{0}$ & 1.3959 \\
\hline & $\mathrm{MgCl}$ & $116.84_{6}$ & $121.67_{6}$ & $120.50_{5}$ & $118.79_{0}$ & 1.3948 \\
\hline & $\mathrm{H}$ & $116.86_{9}$ & $121.66_{3}$ & $120.50_{1}$ & $118.80_{2}$ & 1.3947 \\
\hline & $\mathrm{F}$ & $116.98_{1}$ & $121.60_{0}$ & $120.48_{3}$ & $118.85_{0}$ & 1.3944 \\
\hline & $\mathrm{ClO}_{3}$ & $117.12_{4}$ & $121.51_{8}$ & $120.46_{2}$ & $118.91_{3}$ & 1.3940 \\
\hline & $\mathrm{PH}_{3}^{+}$ & $117.37_{2}$ & $121.37_{4}$ & $120.42_{9}$ & $119.02_{0}$ & 1.3935 \\
\hline
\end{tabular}




\begin{tabular}{|c|c|c|c|c|c|c|}
\hline & $\mathrm{NH}_{3}{ }^{+}$ & $117.41_{7}$ & $121.34_{8}$ & $120.42_{2}$ & $119.04_{0}$ & 1.3934 \\
\hline & $\mathrm{OH}_{2}^{+}$ & $117.49_{5}$ & $121.30_{3}$ & $120.41_{1}$ & $119.07_{4}$ & 1.3933 \\
\hline 3 & $\mathrm{O}^{-}$ & $117.60_{0}$ & $121.34_{8}$ & $120.28_{6}$ & $119.13_{2}$ & 1.3925 \\
\hline & $\mathrm{BH}_{3}^{-}$ & $117.67_{2}$ & $121.30_{6}$ & $120.27_{6}$ & $119.16_{2}$ & 1.3923 \\
\hline & $\mathrm{COO}^{-}$ & $117.79_{4}$ & $121.23_{9}$ & $120.25_{5}$ & $119.21_{6}$ & 1.3919 \\
\hline & $\mathrm{MgCl}$ & $118.15_{9}$ & $121.04_{1}$ & $120.18_{3}$ & $119.39_{1}$ & 1.3907 \\
\hline & $\mathrm{H}$ & $118.20_{1}$ & $121.01_{7}$ & $120.17_{8}$ & $119.40_{8}$ & 1.3906 \\
\hline & $\mathrm{F}$ & $118.30_{9}$ & $120.95_{5}$ & $120.16_{4}$ & $119.45_{2}$ & 1.3904 \\
\hline & $\mathrm{ClO}_{3}$ & $118.47_{3}$ & $120.86_{0}$ & $120.14_{1}$ & $119.52_{4}$ & 1.3900 \\
\hline & $\mathrm{PH}_{3}^{+}$ & $118.73_{2}$ & $120.71_{5}$ & $120.09_{7}$ & $119.64_{4}$ & 1.3895 \\
\hline & $\mathrm{NH}_{3}{ }^{+}$ & $118.77_{0}$ & $120.69_{1}$ & $120.09_{4}$ & $119.65_{9}$ & 1.3895 \\
\hline & $\mathrm{OH}_{2}^{+}$ & $118.85_{4}$ & $120.64_{1}$ & $120.08_{4}$ & $119.69_{5}$ & 1.3894 \\
\hline 4 & $\mathrm{O}^{-}$ & $117.57_{8}$ & $121.35_{0}$ & $120.31_{5}$ & $119.09_{1}$ & 1.3925 \\
\hline & $\mathrm{BH}_{3}^{-}$ & $117.66_{8}$ & $121.30_{5}$ & $120.29_{0}$ & $119.14_{1}$ & 1.3922 \\
\hline & $\mathrm{COO}^{-}$ & $117.81_{1}$ & $121.22_{4}$ & $120.27_{2}$ & $119.19_{5}$ & 1.3917 \\
\hline & $\mathrm{MgCl}$ & $118.11_{9}$ & $121.06_{0}$ & $120.21_{0}$ & $119.33_{9}$ & 1.3906 \\
\hline & $\mathrm{H}$ & $118.17_{5}$ & $121.02_{5}$ & $120.20_{9}$ & $119.35_{4}$ & 1.3904 \\
\hline & $\mathrm{F}$ & $118.32_{5}$ & $120.93_{9}$ & $120.19_{4}$ & $119.40_{7}$ & 1.3901 \\
\hline & $\mathrm{ClO}_{3}$ & $118.46_{8}$ & $120.85_{6}$ & $120.18_{1}$ & $119.45_{9}$ & 1.3896 \\
\hline & $\mathrm{PH}_{3}^{+}$ & $118.69_{5}$ & $120.73_{4}$ & $120.13_{8}$ & $119.56_{0}$ & 1.3891 \\
\hline & $\mathrm{NH}_{3}^{+}$ & $118.75_{6}$ & $120.69_{7}$ & $120.13_{5}$ & $119.58_{0}$ & 1.3889 \\
\hline & $\mathrm{OH}_{2}^{+}$ & $118.85_{6}$ & $120.63_{7}$ & $120.12_{8}$ & $119.61_{2}$ & 1.3887 \\
\hline
\end{tabular}

${ }^{a}$ Bond angles are in degrees, bond distances in $\AA$.

${ }^{b}$ The small intervals of values spanned by some of the ring angles and the correlation introduced by geometrical constraints make it preferable to present bond angles with three decimal figures. Using only two would artificially lower most of the correlation coefficients in Table S4, without otherwise affecting the results of the present study. 
TABLE S2: Selected Geometrical Parameters of the Benzene Ring in Molecular Systems 1-4, from B3LYP/6-311++G** Calculations ${ }^{a, b}$

\begin{tabular}{|c|c|c|c|c|c|c|}
\hline $\begin{array}{l}\text { molecular } \\
\text { system }\end{array}$ & substituent & $\alpha$ & $\beta$ & $\gamma$ & $\delta$ & $a$ \\
\hline \multirow[t]{21}{*}{1} & $\mathrm{O}^{-}$ & $116.31_{1}$ & $122.02_{1}$ & $120.50_{9}$ & $118.62_{8}$ & 1.4053 \\
\hline & $\mathrm{BH}_{3}^{-}$ & $116.36_{3}$ & $121.99_{4}$ & $120.50_{1}$ & $118.64_{8}$ & 1.4049 \\
\hline & $\mathrm{COO}^{-}$ & $116.46_{0}$ & $121.93_{6}$ & $120.48_{8}$ & $118.69_{0}$ & 1.4046 \\
\hline & $\mathrm{MgCl}$ & $116.86_{4}$ & $121.70_{1}$ & $120.42_{3}$ & $118.88_{5}$ & 1.4032 \\
\hline & $\mathrm{H}$ & $116.84_{3}$ & $121.71_{1}$ & $120.43_{0}$ & $118.87_{4}$ & 1.4033 \\
\hline & $\mathrm{Me}$ & $116.86_{0}$ & $121.70_{1}$ & $120.42_{6}$ & $118.88_{4}$ & 1.4033 \\
\hline & $\mathrm{SiH}_{3}$ & $116.88_{5}$ & $121.68_{8}$ & $120.42_{3}$ & $118.89_{3}$ & 1.4032 \\
\hline & $\mathrm{OMe}$ & $116.88_{6}$ & $121.68_{6}$ & $120.42_{3}$ & $118.89_{4}$ & 1.4032 \\
\hline & $\mathrm{NH}_{2}$ & $116.88_{6}$ & $121.68_{7}$ & $120.42_{3}$ & $118.89_{2}$ & 1.4032 \\
\hline & $\mathrm{OH}$ & $116.89_{8}$ & $121.67_{9}$ & $120.41_{9}$ & $118.90_{4}$ & 1.4032 \\
\hline & COMe & $116.90_{6}$ & $121.67_{4}$ & $120.42_{1}$ & $118.90_{3}$ & 1.4031 \\
\hline & $\mathrm{F}$ & $116.93_{8}$ & $121.65_{5}$ & $120.41_{6}$ & $118.91_{9}$ & 1.4031 \\
\hline & $\mathrm{Cl}$ & $116.95_{6}$ & $121.64_{4}$ & $120.41_{4}$ & $118.92_{7}$ & 1.4030 \\
\hline & $\mathrm{CF}_{3}$ & $116.96_{4}$ & $121.63_{9}$ & $120.41_{5}$ & $118.92_{7}$ & 1.4030 \\
\hline & $\mathrm{COCl}$ & $116.99_{0}$ & $121.62_{5}$ & $120.41_{2}$ & $118.93_{6}$ & 1.4029 \\
\hline & $\mathrm{CN}$ & $116.98_{7}$ & $121.62_{5}$ & $120.41_{3}$ & $118.93_{6}$ & 1.4029 \\
\hline & $\mathrm{NO}_{2}$ & $117.00_{0}$ & $121.61_{8}$ & $120.41_{0}$ & $118.94_{3}$ & 1.4029 \\
\hline & $\mathrm{ClO}_{3}$ & $117.11_{1}$ & $121.55_{0}$ & $120.40_{0}$ & $118.98_{8}$ & 1.4026 \\
\hline & $\mathrm{PH}_{3}^{+}$ & $117.39_{5}$ & $121.37_{4}$ & $120.36_{6}$ & $119.12_{4}$ & 1.4021 \\
\hline & $\mathrm{NH}_{3}^{+}$ & $117.40_{8}$ & $121.36_{4}$ & $120.36_{7}$ & $119.12_{7}$ & 1.4022 \\
\hline & $\mathrm{OH}_{2}^{+}$ & $117.51_{7}$ & $121.29_{9}$ & $120.35_{6}$ & $119.17_{0}$ & 1.4021 \\
\hline \multirow[t]{8}{*}{2} & $\mathrm{O}^{-}$ & $116.46_{9}$ & $121.91_{8}$ & $120.51_{2}$ & $118.67_{0}$ & 1.4054 \\
\hline & $\mathrm{BH}_{3}^{-}$ & $116.52_{2}$ & $121.89_{4}$ & $120.49_{8}$ & $118.69_{3}$ & 1.4049 \\
\hline & $\mathrm{COO}^{-}$ & $116.63_{5}$ & $121.82_{9}$ & $120.48_{1}$ & $118.74_{3}$ & 1.4044 \\
\hline & $\mathrm{MgCl}$ & $116.97_{3}$ & $121.63_{2}$ & $120.42_{9}$ & $118.90_{4}$ & 1.4030 \\
\hline & $\mathrm{H}$ & $116.99_{0}$ & $121.62_{2}$ & $120.42_{6}$ & $118.91_{1}$ & 1.4030 \\
\hline & $\mathrm{F}$ & $117.11_{9}$ & $121.54_{8}$ & $120.40_{9}$ & $118.96_{7}$ & 1.4027 \\
\hline & $\mathrm{ClO}_{3}$ & $117.24_{6}$ & $121.47_{2}$ & $120.39_{3}$ & $119.02_{4}$ & 1.4023 \\
\hline & $\mathrm{PH}_{3}^{+}$ & $117.51_{5}$ & $121.30_{3}$ & $120.37_{0}$ & $119.13_{7}$ & 1.4018 \\
\hline
\end{tabular}




\begin{tabular}{|c|c|c|c|c|c|c|}
\hline & $\mathrm{NH}_{3}{ }^{+}$ & $117.54_{1}$ & $121.28_{7}$ & $120.36_{5}$ & $119.15_{3}$ & 1.4018 \\
\hline & $\mathrm{OH}_{2}^{+}$ & $117.63_{5}$ & $121.23_{0}$ & $120.35_{1}$ & $119.20_{0}$ & 1.4018 \\
\hline 3 & $\mathrm{O}^{-}$ & $117.52_{0}$ & $121.41_{7}$ & $120.23_{0}$ & $119.18_{5}$ & 1.4019 \\
\hline & $\mathrm{BH}_{3}^{-}$ & $117.57_{7}$ & $121.38_{2}$ & $120.23_{1}$ & $119.19_{6}$ & 1.4017 \\
\hline & $\mathrm{COO}^{-}$ & $117.72_{0}$ & $121.30_{3}$ & $120.20_{7}$ & $119.25_{8}$ & 1.4011 \\
\hline & $\mathrm{MgCl}$ & $118.11_{2}$ & $121.07_{9}$ & $120.14_{4}$ & $119.44_{0}$ & 1.3997 \\
\hline & $\mathrm{H}$ & $118.16_{4}$ & $121.04_{9}$ & $120.14_{3}$ & $119.45_{0}$ & 1.3995 \\
\hline & $\mathrm{F}$ & $118.26_{1}$ & $120.99_{0}$ & $120.13_{0}$ & $119.49_{7}$ & 1.3993 \\
\hline & $\mathrm{ClO}_{3}$ & $118.42_{2}$ & $120.89_{2}$ & $120.11_{8}$ & $119.55_{6}$ & 1.3990 \\
\hline & $\mathrm{PH}_{3}^{+}$ & $118.73_{6}$ & $120.70_{4}$ & $120.08_{3}$ & $119.69_{0}$ & 1.3984 \\
\hline & $\mathrm{NH}_{3}^{+}$ & $118.75_{2}$ & $120.69_{4}$ & $120.07_{7}$ & $119.70_{6}$ & 1.3985 \\
\hline & $\mathrm{OH}_{2}^{+}$ & $118.83_{8}$ & $120.63_{7}$ & 120.069 & $119.74_{6}$ & 1.3985 \\
\hline 4 & $\mathrm{O}^{-}$ & $117.34_{9}$ & $121.47_{8}$ & $120.32_{4}$ & $119.04_{5}$ & 1.4034 \\
\hline & $\mathrm{BH}_{3}^{-}$ & $117.52_{2}$ & $121.40_{5}$ & $120.24_{8}$ & $119.17_{1}$ & 1.4016 \\
\hline & $\mathrm{COO}^{-}$ & $117.68_{5}$ & $121.31_{6}$ & $120.22_{8}$ & $119.22_{5}$ & 1.4010 \\
\hline & $\mathrm{MgCl}$ & $118.03_{9}$ & $121.11_{9}$ & $120.16_{9}$ & $119.38_{3}$ & 1.3995 \\
\hline & $\mathrm{H}$ & $118.04_{5}$ & $121.09_{2}$ & $120.20_{3}$ & $119.36_{1}$ & 1.3996 \\
\hline & $\mathrm{F}$ & $118.22_{9}$ & $121.00_{1}$ & $120.17_{0}$ & $119.42_{7}$ & 1.3990 \\
\hline & $\mathrm{ClO}_{3}$ & $118.41_{1}$ & $120.89_{5}$ & $120.15_{2}$ & $119.49_{4}$ & 1.3986 \\
\hline & $\mathrm{PH}_{3}^{+}$ & $118.68_{4}$ & $120.72_{2}$ & $120.13_{6}$ & $119.59_{8}$ & 1.3979 \\
\hline & $\mathrm{NH}_{3}^{+}$ & $118.71_{8}$ & $120.70_{6}$ & $120.12_{3}$ & $119.62_{3}$ & 1.3979 \\
\hline & $\mathrm{OH}_{2}^{+}$ & $118.86_{3}$ & $120.61_{0}$ & $120.12_{2}$ & $119.67_{1}$ & 1.3976 \\
\hline
\end{tabular}

${ }^{a}$ Bond angles are in degrees, bond distances in $\AA$.

${ }^{b}$ The small intervals of values spanned by some of the ring angles and the correlation introduced by geometrical constraints make it preferable to present bond angles with three decimal figures. Using only two would artificially lower most of the correlation coefficients in Table 1, without otherwise affecting the results of the present study. 
TABLE S3: Univariate Statistics for the Internal and Angular Symmetry Coordinates of the Benzene Ring in $\mathrm{Ph}-\mathrm{C}\left(\mathrm{CH}_{2}-\mathrm{CH}_{2}\right)_{3} \mathrm{C}-\mathrm{X}$ Molecules, from B3LYP/6-311++G** Calculations

\begin{tabular}{|c|c|c|c|c|}
\hline parameter & $\begin{array}{l}\text { minimum } \\
\text { value }^{a}\end{array}$ & $\begin{array}{l}\operatorname{maximum} \\
\text { value }^{a}\end{array}$ & mean $^{a}$ & variance $^{b}$ \\
\hline$\alpha$ & 116.31 & 117.52 & 116.93 & 0.0893 \\
\hline$\beta$ & 121.30 & 122.02 & 121.66 & 0.0322 \\
\hline$\gamma$ & 120.36 & 120.51 & 120.42 & 0.0015 \\
\hline$\delta$ & 118.63 & 119.17 & 118.91 & 0.0185 \\
\hline$a$ & 1.4021 & 1.4053 & 1.4032 & $671 \times 10^{-9}$ \\
\hline$b$ & 1.3935 & 1.3944 & 1.3939 & $44 \times 10^{-9}$ \\
\hline$c$ & 1.3916 & 1.3931 & 1.3921 & $163 \times 10^{-9}$ \\
\hline$D_{4}$ & -4.38 & -2.87 & -3.61 & 0.1418 \\
\hline$D_{5}$ & -0.46 & -0.41 & -0.43 & 0.0002 \\
\hline$D_{6}$ & -2.18 & -1.44 & -1.82 & 0.0331 \\
\hline
\end{tabular}

${ }^{a}$ Values for angles and angular symmetry coordinates are in deg, those for distances in A.

${ }^{b}$ Values for angles and angular symmetry coordinates are in $\mathrm{deg}^{2}$, those for distances in $\AA^{2}$. 
TABLE S4: Linear Regressions Between Angular Parameters of the Benzene Ring in Molecular Systems 1-4, from HF/6-31G* Calculations

\begin{tabular}{|c|c|c|c|c|}
\hline regression ${ }^{a}$ & $\begin{array}{c}\text { molecular } \\
\text { system }\end{array}$ & $\begin{array}{l}\text { angular } \\
\text { coefficient }\end{array}$ & intercept (deg) & $\begin{array}{l}\text { correlation } \\
\text { coefficient }\end{array}$ \\
\hline \multirow[t]{4}{*}{$\Delta \beta$ vs $\Delta \alpha$} & 1 & $-0.569(2)$ & $-0.117(7)$ & -0.9999 \\
\hline & 2 & $-0.566(2)$ & $-0.111(7)$ & -0.9999 \\
\hline & 3 & $-0.561(3)$ & $0.002(5)$ & -0.9999 \\
\hline & 4 & $-0.558(3)$ & $0.003(5)$ & -0.9999 \\
\hline \multirow[t]{4}{*}{$\Delta \gamma$ vs $\Delta \alpha$} & 1 & $-0.164(3)$ & $-0.032(11)$ & -0.9961 \\
\hline & 2 & $-0.159(4)$ & $0.008(11)$ & -0.9980 \\
\hline & 3 & $-0.164(4)$ & $-0.110(7)$ & -0.9979 \\
\hline & 4 & $-0.144(6)$ & $-0.046(11)$ & -0.9926 \\
\hline \multirow[t]{4}{*}{$\Delta \delta$ vs $\Delta \alpha$} & 1 & $0.464(3)$ & $0.290(9)$ & 0.9997 \\
\hline & 2 & $0.447(2)$ & $0.196(8)$ & 0.9999 \\
\hline & 3 & $0.451(3)$ & $0.215(5)$ & 0.9999 \\
\hline & 4 & $0.406(6)$ & $0.086(12)$ & 0.9990 \\
\hline \multirow[t]{4}{*}{$D_{4}$ vs $D_{6}$} & 1 & $2.30(3)$ & $0.36(5)$ & 0.9988 \\
\hline & 2 & $2.25(3)$ & $0.13(5)$ & 0.9994 \\
\hline & 3 & $2.29(3)$ & $0.60(3)$ & 0.9993 \\
\hline & 4 & $2.09(5)$ & $0.23(5)$ & 0.9981 \\
\hline
\end{tabular}

${ }^{a}$ The number of data points is 21 for molecular system $\mathbf{1}, 10$ for $\mathbf{2}, \mathbf{3}$, and $\mathbf{4}$. 
TABLE S5: Univariate Statistics for the Internal Coordinates of the

Bicyclo[2.2.2] octane Cage in $\mathrm{Ph}-\mathrm{C}\left(\mathrm{CH}_{2}-\mathrm{CH}_{2}\right)_{3} \mathrm{C}-\mathrm{X}$ Molecules, from B3LYP/6311++G** Calculations

\begin{tabular}{|c|c|c|c|c|}
\hline parameter & $\begin{array}{c}\text { minimum } \\
\text { value }^{a}\end{array}$ & $\begin{array}{c}\text { value }^{a} \\
\text { maximum }\end{array}$ & mean $^{a}$ & variance $^{b}$ \\
\hline$\alpha^{\prime}$ & 105.44 & 113.03 & 109.02 & 2.744 \\
\hline$\beta^{\prime}$ & 105.55 & 113.47 & 109.89 & 3.226 \\
\hline$\gamma^{\prime}$ & 110.84 & 111.67 & 111.26 & 0.048 \\
\hline$\delta^{\prime}$ & 107.46 & 107.78 & 107.60 & 0.006 \\
\hline$a^{\prime}$ & 1.5065 & 1.5711 & 1.5383 & $177 \times 10^{-6}$ \\
\hline$b^{\prime}$ & 1.5536 & 1.5716 & 1.5577 & $20 \times 10^{-6}$ \\
\hline$c^{\prime}$ & 1.5505 & 1.5549 & 1.5525 & $1 \times 10^{-6}$ \\
\hline$r(\mathrm{C} \cdots \mathrm{C})$ & 2.5392 & 2.7371 & 2.6441 & $1918 \times 10^{-6}$ \\
\hline
\end{tabular}


TABLE S6: Selected Geometrical Parameters of the Bicyclo[2.2.2]octane Cage in $\mathrm{Ph}-\mathrm{C}\left(\mathrm{CH}_{2}-\mathrm{CH}_{2}\right)_{3} \mathrm{C}-\mathrm{X}$ Molecules, from HF/6-31G* Calculations ${ }^{a}$

\begin{tabular}{|c|c|c|c|c|}
\hline substituent & $\alpha^{\prime}$ & $\beta^{\prime}$ & $a^{\prime}$ & $r(\mathrm{C} \cdots \mathrm{C})$ \\
\hline $\mathrm{O}^{-}$ & 105.51 & 113.51 & 1.5602 & 2.7243 \\
\hline $\mathrm{BH}_{3}^{-}$ & 106.84 & 112.46 & 1.5388 & 2.6941 \\
\hline $\mathrm{COO}^{-}$ & 107.98 & 111.17 & 1.5342 & 2.6630 \\
\hline $\mathrm{MgCl}$ & 107.38 & 111.61 & 1.5433 & 2.6806 \\
\hline $\mathrm{H}$ & 109.00 & 110.06 & 1.5310 & 2.6337 \\
\hline $\mathrm{Me}$ & 108.01 & 111.08 & 1.5358 & 2.6595 \\
\hline $\mathrm{SiH}_{3}$ & 107.97 & 110.90 & 1.5422 & 2.6630 \\
\hline $\mathrm{OMe}$ & 108.68 & 110.42 & 1.5303 & 2.6420 \\
\hline $\mathrm{NH}_{2}$ & 108.27 & 110.87 & 1.5329 & 2.6515 \\
\hline $\mathrm{OH}$ & 108.82 & 110.38 & 1.5274 & 2.6365 \\
\hline $\mathrm{COMe}$ & 108.37 & 110.46 & 1.5391 & 2.6522 \\
\hline $\mathrm{F}$ & 109.89 & 109.30 & 1.5196 & 2.6077 \\
\hline $\mathrm{Cl}$ & 109.74 & 109.21 & 1.5256 & 2.6157 \\
\hline $\mathrm{CF}_{3}$ & 108.82 & 109.98 & 1.5370 & 2.6393 \\
\hline $\mathrm{COCl}$ & 108.81 & 109.89 & 1.5388 & 2.6407 \\
\hline $\mathrm{CN}$ & 108.99 & 109.73 & 1.5388 & 2.6342 \\
\hline $\mathrm{NO}_{2}$ & 109.90 & 108.84 & 1.5291 & 2.6115 \\
\hline $\mathrm{ClO}_{3}$ & 111.19 & 107.34 & 1.5232 & 2.5783 \\
\hline $\mathrm{PH}_{3}^{+}$ & 109.67 & 108.76 & 1.5419 & 2.6190 \\
\hline $\mathrm{NH}_{3}^{+}$ & 110.47 & 108.33 & 1.5246 & 2.5946 \\
\hline $\mathrm{OH}_{2}^{+}$ & 112.54 & 106.05 & 1.5086 & 2.5408 \\
\hline
\end{tabular}


TABLE S7: Selected Geometrical Parameters of the Bicyclo[2.2.2]octane Cage in $\mathrm{Ph}-\mathrm{C}\left(\mathrm{CH}_{2}-\mathrm{CH}_{2}\right)_{3} \mathrm{C}-\mathrm{X}$ Molecules, from B3LYP/6-311++G** Calculations $^{a}$

\begin{tabular}{|c|c|c|c|c|}
\hline substituent & $\alpha^{\prime}$ & $\beta^{\prime}$ & $a^{\prime}$ & $r(\mathrm{C} \cdots \mathrm{C})$ \\
\hline $\mathrm{O}^{-}$ & 105.44 & 113.47 & 1.5711 & 2.7371 \\
\hline $\mathrm{BH}_{3}^{-}$ & 106.87 & 112.51 & 1.5430 & 2.7037 \\
\hline $\mathrm{COO}^{-}$ & 107.98 & 111.22 & 1.5395 & 2.6736 \\
\hline $\mathrm{MgCl}$ & 107.75 & 111.26 & 1.5463 & 2.6822 \\
\hline $\mathrm{H}$ & 109.04 & 110.02 & 1.5365 & 2.6425 \\
\hline $\mathrm{Me}$ & 107.97 & 111.13 & 1.5420 & 2.6695 \\
\hline $\mathrm{SiH}_{3}$ & 108.11 & 110.78 & 1.5469 & 2.6696 \\
\hline $\mathrm{OMe}$ & 108.74 & 110.34 & 1.5363 & 2.6500 \\
\hline $\mathrm{NH}_{2}$ & 108.26 & 110.87 & 1.5396 & 2.6614 \\
\hline $\mathrm{OH}$ & 108.91 & 110.28 & 1.5335 & 2.6438 \\
\hline $\mathrm{COMe}$ & 108.37 & 110.45 & 1.5456 & 2.6622 \\
\hline $\mathrm{F}$ & 110.10 & 109.06 & 1.5245 & 2.6129 \\
\hline $\mathrm{Cl}$ & 109.90 & 109.08 & 1.5291 & 2.6214 \\
\hline $\mathrm{CF}_{3}$ & 108.85 & 109.89 & 1.5438 & 2.6484 \\
\hline $\mathrm{COCl}$ & 108.75 & 109.90 & 1.5468 & 2.6517 \\
\hline $\mathrm{CN}$ & 108.82 & 109.84 & 1.5486 & 2.6483 \\
\hline $\mathrm{NO}_{2}$ & 109.95 & 108.85 & 1.5333 & 2.6197 \\
\hline $\mathrm{ClO}_{3}$ & 112.12 & 106.52 & 1.5155 & 2.5638 \\
\hline $\mathrm{PH}_{3}^{+}$ & 109.92 & 108.46 & 1.5468 & 2.6222 \\
\hline $\mathrm{NH}_{3}^{+}$ & 110.53 & 108.29 & 1.5294 & 2.6025 \\
\hline $\mathrm{OH}_{2}^{+}$ & 113.03 & 105.55 & 1.5065 & 2.5392 \\
\hline
\end{tabular}

